\begin{abstract}
Packet level measurement is now routinely used to evaluate the loss and delay performance of broadband networks. In active measurement, probe packets provide samples of the loss and delay, and from these samples the performance of the traffic as a whole can be deduced. However this is prone to errors: inaccuracy due to taking insufficient samples, self-interference due to injecting too many probe packets, and possible sample-correlation induced bias. In this paper we consider the optimization of probing rate by treating all measurements as numerical experiments which can be optimally designed by using the statistical principles of Design of Experiments. We develop an analytical technique that quantifies an overall utility function associated with: i) the disruption caused per probe packet, ii) the bias and iii) the variance as a function of the probing (sampling) rate. Our numerical results show that the optimal probing rate depends strongly on what parameter the network engineer seeks to measure.
\end{abstract}




\title{
A utility based framework for optimal network measurement
}

\author{
B.M.Parker and S.G.Gilmour \\ Southampton Statistical Sciences Research Institute, \\ University of Southampton, UK \\ J.Schormans \\ School of Electronic Engineering and Computer Sciences, \\ Queen Mary University of London, United Kingdom
}

September 30, 2013

\section{Introduction}

Recent research into broadband packet networks has considered the injection of probe packets to measure the performance of the network; for example whether it is best to probe at a uniform rate, or to send probes according to some renewal process, such as a Poisson process. In general this research on probing has focused on queueing systems as good general models of packet level network performance. Whilst modeling a large network may be impossible, by representing the network as a queue or a series of queues, the problem becomes more tractable.

The measurement of packet networks has a number of possible moti- 
vations: to provide solid numerical support of the guarantees written into service level agreements, and to fault-find are two that are not necessarily real time. Probing results are also used in support of measurement based admission control (MBAC). In MBAC schemes the network state must be rapidly evaluated such that the decision to admit (or not) a new connection can be made quickly. Recently the main focus has been on wireless systems. In [1], the authors discuss the measurement implications for end-to-end applications with Quality of Service (QoS) requirements, and propose a new tool for measurement purposes, while in [2] and [3] wireless MBAC systems are proposed.

Crucially we regard our network measurement process as a numerical experiment we wish to measure; we appeal to methodology from the statistical theory of design of experiments to apply these principles to the measurement of packet networks. See [4] for a good introduction to optimal experimental design.

In previous work [5], we have discussed some approaches for optimally designing experiments to measure networks. In that paper, for tractability we considered networks where we knew a great deal about the performance of the network element under study. While this earlier approach yielded optimal probing rates for certain simple (i.e. single buffer) schemes, it would be hard to use for larger networks.

In measuring packet networks through active probing, we argue that the three main objectives in any measurement are to measure the network perfor- 
mance: 1) accurately; 2) precisely; and 3) whilst causing minimal disruption to the underlying user traffic.

In statistical terms, we are concerned with finding an estimator for some property of the network we wish to measure. Recall that an estimator is a function which takes some observable data and produces an estimate of an unknown parameter we are interested in measuring. An estimate is a particular value of this estimator, given some particular data. We seek to minimize: 1) the bias of the estimator; 2) the variance of the estimator; 3) some measure of disruption caused by active probing which we shall define later. We argue in this paper that all network measurement algorithms seek to accomplish some of these three aims, either implicitly or explicitly.

We begin in section 2 by reviewing previous work on measurement of networks. We first consider previous work on network measurement using inference from partial information about queues, such as that obtained by active probing. We then look at how we can use statistical principles of design of experiments to find an optimal active probing rate. In section 3 we develop a general utility based methodology that can be widely applied to any packet network given only some key parameters. We present a series of examples of the use of the methodology in section 4 . We conclude in section 5. 


\section{Related work}

There is a significant body of research predominantly concerned with devising probabilistic methods and sometimes using them in model-based prediction, rather than measurement-based inference, which we do not consider here. Instead, we review research on inference about queues from data gathered from customers within those queues.

\subsection{Inference}

Clarke [6] first investigated statistical inference in queues, deriving formulae for maximum likelihood estimators (MLEs) for the M/M/1 queue. He chose this particular queue with one particular sampling method (in which we begin observing the queue at time zero with an initial number of packets $\nu=n(0)$,

$\left.\nu \sim g e o\left(\frac{\lambda}{\mu}\right)\right)$. The exact sampling frame, and initial distribution of the queue, are sensible, yet arbitrary, but the method for calculating the MLE was new and useful.

Jenkins[7] compared relative efficiencies of the direct estimate for the mean waiting time with that suggested by Clarke, and concludes that the MLE has a lower asymptotic variance, particularly for high values of load.

Aigner[8] summarizes work at the time (1974) and compares various estimators for arrival rate and departure rate parameters in an $\mathrm{M} / \mathrm{M} / 1$ queue, in which the number of packets sampled is fixed. There are a vast number of estimators (e.g. MLE, least squares) even for this simple setup; Aigner 
uses the asymptotic variance of the different estimators as the criterion to decide which is best. He notes that this is a somewhat arbitrary optimality criterion, does not apply to inference from small samples, and does not take any account of the time needed to gather these data. However, Aigner does clearly indicate the difficulty in determining, even for a fixed sampling method and a simple queue, which estimator is the best.

Reynolds[9] looks at covariance structure in queues, and in particular (section 5) assesses variances of different estimators. His results are presented perhaps less methodically than Aigner, but they are more rigorous and not limited only to $\mathrm{M} / \mathrm{M} / 1$ queues. The sampling frames used are also different, as Reynolds observes the queues up to some fixed time whereas Aigner looks at a fixed number of customers.

Basawa and Prahu[10] prove that MLEs are asymptotically normally distributed, and show how this can be used for the example of an M/M/1 queue. This work draws on probabilistic results from Billingsley[11]. They later[12] derive MLEs and Fisher information for queues whose arrival and departure distributions come from exponential families, so that two parameters are to be estimated, a general model with much relevance to most queues studied.

Achaya[13] extends work in [12] by showing how quickly MLEs converge; in other words, how big a sample is needed for the asymptotic theory developed to apply.

In a later paper, Basawa et al.[14] have also tried to establish a general framework to find the Fisher information matrix, which is useful in calculat- 
ing designs for experiments, and was also used by us in [5].

Most of this research assumes that the number of samples tends to infinity, i.e. that we have an unlimited amount of time in which to measure the network. For example, we see in [14] that the MLE is not affected by the choice of sampling method, although it does not follow that what is best for a large sample is best for a small experiment. Indeed, in most network measurement research, there is an implicit stationarity assumption, as described by Roughan[15]: we consider a network where the traffic rate does not change, in other words that we are looking over a short enough period of time that this assumption is valid. As estimators are used on a small number of data gathered in a short period of time, the asymptotic results cannot be relied on.

A summary of this previous research would be that estimators of queues can be shown to be sensible given that we have a long time to observe the queue, but little is known theoretically about how well measurements can be taken on queues over a short measurement period. The implication of this is that in practice the best estimators for a given problem are not necessarily being used by practitioners, even if they are aware of the theory.

\subsection{Partial Information}

In a real measurement experiment, for example when considering active probing, the experimenter is limited in the knowledge he is able to gather. He does not have access to the underlying (cross-traffic) packets in a system. 
Basawa et al.[14] also look at the interesting problem of finding MLEs given only partial information, here considering estimation given only waiting time data. They show asymptotic consistency and normality of the estimators, and present forms for the MLE and Fisher information for partial information in the special cases of $\mathrm{M} / E_{k} / 1$, where the service times have the Erlangian distribution, and of $\mathrm{M} / \mathrm{M} / 1$. The analytical results show that these MLEs turn out to be rather poor and are biased.

Basawa[16] develops this work further, looking at queues where both service and inter-arrival times are drawn from exponential families, when only the sample packet's waiting or system time, together with queue idle times, are known.

Chen[17] takes an M/D/1 queue for which we only have knowledge of waiting and service times for some packets, and tries to find the MLE for the arrival rate $\lambda$ for $k$ observed packets. We could say that some packets are controlled by the experimenter, but most are not controllable. Based on the partial data available, a complex form for the MLE is derived, and Chen proves that the distribution of the MLE is asymptotically normal. He concludes that the method is more generally applicable, although the exact method to be used will vary depending on what data are available, and what queue is being measured.

None of this research explicitly considers active probing, where extra packets are put into the system; instead, information on a random sample of packets is known. The authors therefore do not need to consider that 
introducing probe packets into the network may cause interference with the data packets; we found this self-interference to be important in [5] and we consider it further in this paper.

\subsection{Recent work on probing packet networks}

The PASTA (Poisson Arrivals See Time Averages) theorem, first formalized by Wolff[18], has been a widely used principle in packet probing; it tells us that if we introduce probe packets according to a Poisson process, then the mean of their waiting time is an unbiased estimator of the waiting time of all packets (combining both probe and underlying data packets) in the queue.

Although the PASTA property is desirable, Roughan[15] compares uniform and Poisson sampling and seeks to explain to a practitioner that both have desirable properties depending on what information he is trying to gather when probing. Baccelli et al.[19] show that, in the case where probes are non intrusive, there is a wider group of mixing processes (they call these 'NIMASTA'), including Poisson, which allow us to 'see time averages', and that some of these have better properties with respect to other measures, such as reducing variance. In the active probing case, where probes are intrusive, they argue that a substantial problem is the 'inversion problem'; i.e. being able to measure waiting time for probe packets does not allow us to infer the waiting time of non-probe packets without error. They call this bias introduced in the estimate by active probing the "inversion bias", and show that Poisson probing does nothing to minimize this. 
They go on to show in [20] that probes introduced with inter-arrival times following a gamma distribution have the lowest mean square error in estimating both delay and packet loss, amongst all queues with convex autocovariance.

Roughan[21] has shown that there are fundamental bounds on how accurately network measurements can be made: that no matter how many active probes are used in a time interval, there is a limit to the knowledge we can gather about a queue. He makes an analogy to Heisenberg's uncertainty principle in quantum mechanics, where our certainty on position or momentum of a sub-atomic particle cannot be increased above a certain limit no matter how many times we observe it. Although his analytic results focus on measurement of a system where we have 'perfect measurements', he generalizes the work to active probing, although he notes that analytic results would be complex in form and derivation.

In [22] Baccelli et al describe recent work in which they argue that backto-back packet trains (recommended for probing by some authors) introduces more bias and delay than using single probe packets. In [23] Sun and Xiao approach optimal delay and loss estimation by using an equivalent random system, and treating it as a linear estimation problem. In [24] Duffield et al. have patented a technique for optimal combination of sampled measurements; however this relates to using optimally placed combinations of router locations. 


\section{Methodology}

We observe in this previous work that there has been some research into finding appropriate estimators of parameters for a network; this largely consists of estimating parameters in known queues, and there has been little research for more general networks. Recent work by Roughan[21] and Baccelli et al. [19],[20] has begun to consider the problem of reducing variance in estimators, but has not really considered the important question of what the optimal probing rate is, or how to find one. We are unaware of research that explicitly covers how active measurement disrupts a network, although minimizing disruption to users is well understood by network engineers, and research is ongoing into QoS[25].

Motivated by this previous work, we now consider the problem of determining an optimal rate at which to probe when considering intrusive probes in a simplified network scenario.

In this paper we view all network measurements as numerical experiments, in which random processes are sampled, and the effectiveness of the sampling is measured by a utility we place on bias and variance in the resulting estimator. In this way we are then able to apply the statistical principles of Design of Experiments (DOE) to network measurement experiments to develop a methodology which enables us to find an optimal active probing rate. We present a general utility function that combines the bias and variance of the estimator with the added congestion in the network caused by 
probing.

\subsection{Model overview and notation}

Suppose we have a network into which packets arrive at a rate $\lambda$, which is fixed but unknown, and in which they are served and leave the system at a rate $\mu$, which is fixed and known.

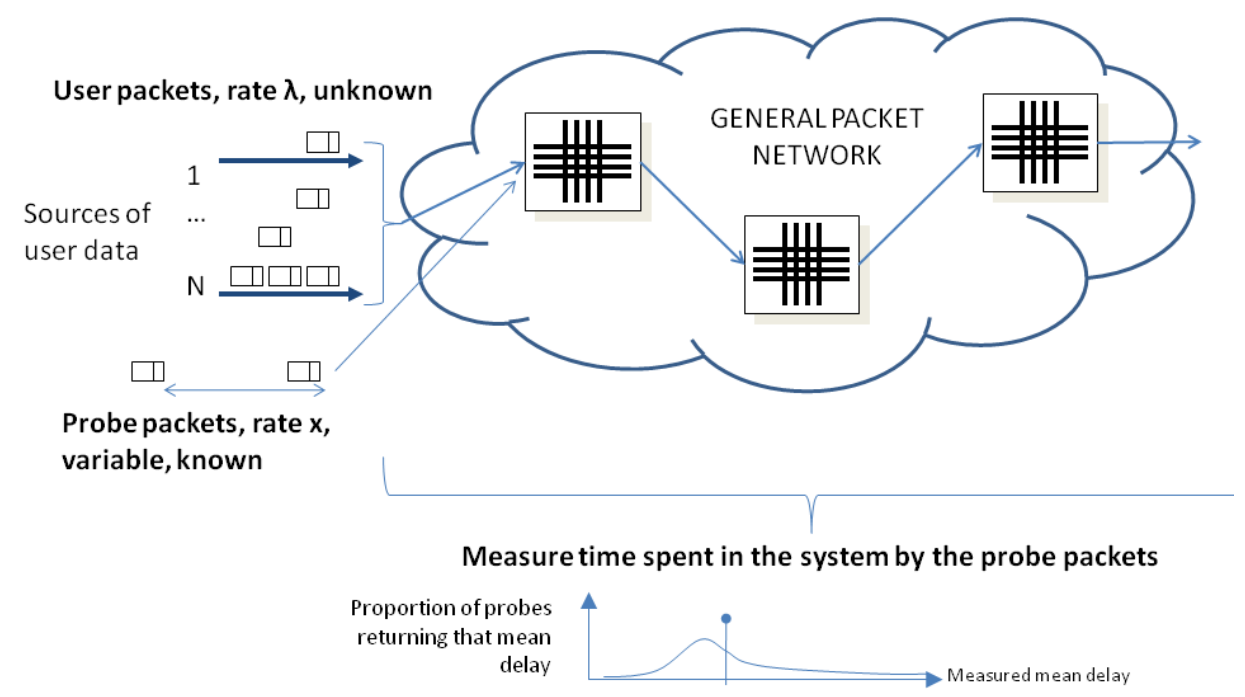

Figure 1: Overview of probing experiment

We wish to find the value of some unknown parameter of the network $\theta$, such as the probability of packet loss or the mean system time for the crosstraffic (user) packets. We assume we are unable to measure this $\theta$ directly, but we instead introduce probe packets into the system at a rate $x$, and monitor when these probes enter the system and when they emerge. We can then make inference about $\theta$ from the amount of time that the probe packets 
spend in the system. Our goal is to find the value of $x$ which allows us to best estimate $\theta$. See Figure 1.

We define $S(t)$ as the amount of time required for all packets in the network at time $t$ to complete service and exit the network. We call this the virtual waiting time, because an imagined packet arriving at time $t$ would spend a time $S(t)$ waiting in the network.

This is a continuous-time right-continuous process which takes non-negative values. Instantaneous jumps occur when a packet enters the system at arrival times $a_{0}, a_{1}, a_{2}, \ldots$ The jumps have magnitude that varies depending on the queue discipline and corresponds to service duration for the packet arriving at that arrival time. For example, in the M/M/1 queue the magnitude of the jumps correspond to the service times and are thus exponentially distributed with parameter $\mu$.

The jump times, $a_{i}$, and the magnitude of the jumps of $S(t)$ are random variables, but otherwise the process is deterministic, changing at rate -1 (decreasing) until it reaches 0 , where it remains until an arrival occurs. Unless we have full knowledge of the queue, we cannot observe $S(t)$ directly, but we make inference about it by introducing $N$ probe packets at times $\tau_{1}, \tau_{2}, \ldots, \tau_{N}$. By introducing new packets into the system, we form a new process $S^{*}(t)$. This is represented diagrammatically in Figure 2 .

We let the number of probes generated by the probing process be $N$ as above (note that $N$ is in general a random variable, but we may fix it, for example by having a fixed time between probes). We denote the time 


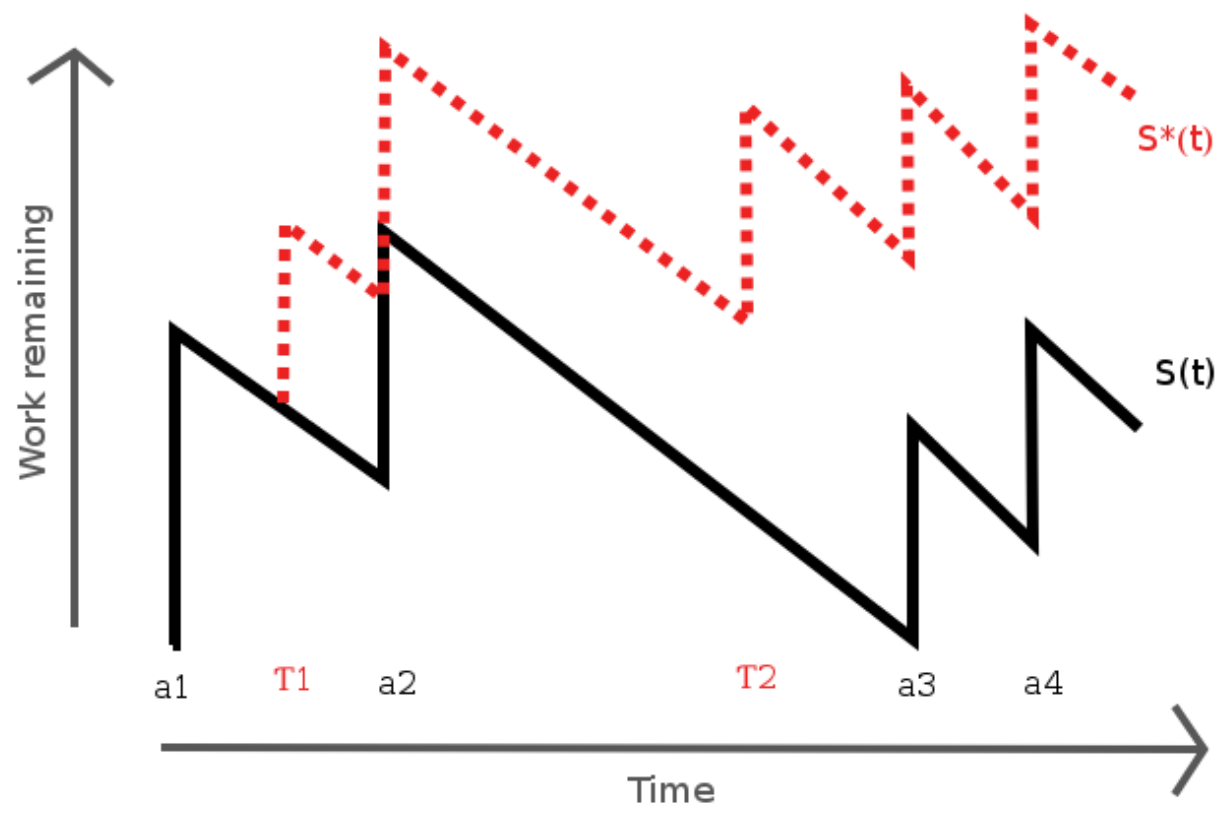

Figure 2: $S(t)$ and $S^{*}(t)$ : Underlying and observed virtual waiting time process 
between probe packet $j$ entering and leaving the system as $y_{j}$, i.e. the system time. We are concerned with $\hat{\lambda}$, an estimate of $\lambda$. Given $N=n$ packets, we have $\tau_{1}<\tau_{2}<\ldots<\tau_{n}$ and observe $S^{*}\left(\tau_{1}\right), S^{*}\left(\tau_{2}\right), \ldots, S^{*}\left(\tau_{n}\right)$ without error. We let $y_{i}=S^{*}\left(\tau_{i}\right)$, and our data are thus $y_{1}, \ldots, y_{n}$, which we may collectively write as the vector $\boldsymbol{y}$.

\subsection{The difficulties with an analytic approach}

Ideally, we wish to find an analytical method of estimating the virtual waiting time, given that we know what it is at certain time points (the probe arrival times). If we can find an analytical function for the evolution of the virtual waiting time under probing, then this will help us in finding exact expressions for the expectation and variance of any estimator under a particular probing pattern.

In other words, we seek to find $P\left[S(t)=y \mid S(0)=y_{0}\right]$. Takacs[26] proves that

$$
\begin{gathered}
\left.P\left[S(t)=y \mid S(0)=y_{0}\right]=P\left(\chi(t) \leq t+y-y_{0}\right)\right)- \\
\int_{0 \leq v \leq z \leq t-y_{0}} \frac{t-z}{t-v} P(\chi(v) \leq v+y, \chi(t) \leq z+y) d v d z
\end{gathered}
$$

for all $y, y_{0} \geq 0$ and $t>0$ where $\chi(t)$ is the total accumulated service time of all customers arriving in $[0, t]$.

If we know the distribution of the service time for a particular queue, then we can evaluate equation (1) to get an explicit form for our virtual waiting 
time function. Chen[17] showed how to do this for an M/D/1 queue. He forms an approximation to $S(t)$ by a random walk

$$
X_{n}=S(n \Delta t)=\max \left(X_{n-1}+Z_{n}, 0\right),
$$

where $Z_{n}$ are I.I.D and represent the combined service time of all arrivals in interval $\Delta t$. Chen forms the recurrence

$$
H_{n}(x)=\int_{0}^{\infty} H_{n-1}(v) H(x-v) d v, \quad x \geq 0
$$

where $H(t)=e^{-\mu v}$ is the PDF for service time as before. The PDF for service time in the M/D/1 case is trivial as service times for all packets are constant, so it is relatively easy to solve this recurrence relation; Chen does this by applying Laplace transforms. For more general networks, $H(t)$ is more complicated, Laplace transforms are not known, and this analytical approach breaks down.

Cox and Isham[27] also considered the problem of determining waiting time given partial information, and did not find it possible, apart from in some specific cases, to derive an analytical virtual waiting time through Laplace transforms. Without a PDF for the virtual waiting time, the design problem of finding an optimal probing rate is also analytically intractable by this method. 


\subsection{Specifying a utility function}

As the analytic approach does not seem tractable for general networks, we consider a utility-based approach to our problem of optimizing probing rates.

We must first consider what we wish to achieve in measuring a network. It is clear that we wish to measure our unknown network parameter (e.g. mean delay, packet loss probability, etc.) accurately. In other words, we wish

to minimize the bias of some estimator $\hat{\theta}$, an estimator formed from some function of $\boldsymbol{Y}, x$, and $\mu$. The bias of an estimator is the difference between the expected value of the estimator and its true value: $\operatorname{bias}(\hat{\theta})=\mathbb{E}(\hat{\theta})-\theta$.

The statistical theory of Design of Experiments also tells us that minimizing the variance of the estimator $\hat{\theta}$ is desirable. Ideally we would like an estimator which is both accurate (low bias) and precise (low variance).

In general, forming an estimate from more data leads to an estimator with lower variance. However to get more data we must probe more. We know that more probes in a network may disrupt the transmission of cross-traffic packets, and we also wish to minimize the disruption of these cross-traffic packets. We measure this disruption per packet at probe rate $x$ as

$$
D(x)=\frac{1}{N}\left(\sum_{i=1}^{N}\left[c\left(S^{*}\left(a_{i}\right)-S\left(a_{i}\right)\right)\right]^{r}\right)^{1 / r}
$$

where $c(w)$ is some cost function for a delay of one packet by an amount $w$, and $r>0$. In general the underlying $S\left(a_{i}\right)$ will be impossible to observe, and we estimate this by simulation. The index $r$ in the disruption function allows 
us to penalize deviations from the mean delay time non-linearly; for example in a VoIP system it may be more damaging to have one packet not delayed and one delayed by $20 \mathrm{~ms}$, rather than having two packets each delayed by 10ms. In an engineering context, $r$ allows us to penalize jitter. $r=1$ corresponds to being ambivalent about jitter, where increasing $r$ means that we penalize high jitter more severely for equal average delays. $0<r<1$ corresponds to penalizing low jitter, although we do not know of a useful application for this.

In order to simultaneously minimize bias, variance, and disruption, we form a general utility function

$$
\psi(\operatorname{Bias}(\hat{\lambda} \mid x), \operatorname{Var}(\hat{(\lambda)} \mid x), D(x)),
$$

and use this to find $x_{\lambda}=\arg \min _{x} \psi(x)$, our optimal probing rate.

The exact form of the utility function will depend on how much we wish to trade accuracy and precision when estimating $\lambda$ compared with the disruption caused when measuring at this probing rate. When combining bias and variance, a natural metric is the mean square error, $\operatorname{MSE}(\hat{\lambda})=[\operatorname{Bias}(\hat{\lambda})]^{2}+$ $\operatorname{Var}(\hat{\lambda})$. This metric is frequently used, and is natural in the sense that it is dimensionally consistent, as Bias ${ }^{2}$, variance, and thus mean square error have units of $s^{-2}$ here. We will work with $\sqrt{\mathrm{MSE}}$, the root mean square error, or RMSE. How much to penalize disruption is more subjective, and will depend on the network under study and the experimenter's view on 
the relative merits of good estimates versus disruption. For example, in a network carrying electronic mail (e.g. SMTP) traffic it may be acceptable to have a delay of several seconds or even minutes, whereas in a network carrying live voice traffic (e.g. VoIP), or electronic trading data, delays of even tenths of a second may have a significant impact on service. We present some examples below.

In order to balance, variance, and disruption, we propose a general form of the utility function

$$
\psi(x)=-[\kappa \sqrt{\operatorname{MSE}(\hat{\lambda})}+(1-\kappa) D(x)]
$$

where the disruption $D(x)$ is defined as equation (4) above, and $0 \leq \kappa \leq 1$. This framework will suit many applications as we demonstrate through examples, although other functions may be useful in particular circumstances.

We could equally well define, for example, a multiplicative utility function, e.g. $\psi_{*}(x)=-\kappa \sqrt{\operatorname{MSE}(\hat{\lambda})}(1-\kappa) D(x)$, however, we feel the additive function is more justified as a probing rate that provided good estimates $(\sqrt{\operatorname{MSE}(\hat{\lambda})}=$ 0) but high disruption, or vice versa, meaning that $\psi_{*}(x)=0$, would not be in line with the goals of the experiment in balancing bias, variance, and disruption. In this paper, we set out to provide a general framework for balancing this goals, and demonstrate through focus on a particular utility function, equation (6), rather than to make a comparison between them.

The choice of $\kappa$, and indeed $r$, in our utility function 6 allows great 
flexibility within our proposed utility function form that penalises disruption, bias, and variance. The choice of $\kappa$ is important, and may require some careful thought (or simulation; see section 4.4) from the engineer conducting measurement to determine it; in short $\kappa$ balances the importance of the measurement and reporting goals against the monetary or other performance cost caused by disruption. If we are unconcerned with disruption caused by measurement, we choose $\kappa=1$, and if we are unconcerned with measurement accuracy we choose $\kappa=0$. For most practical applications, we will choose $\kappa$ between 0 and 1 , see section 4.4 for some examples.

The utility function we propose is without a unit, in the sense that it is a function which maps from a two dimensional domain (of RMSE combined with disruption) to a one dimensional range (utility). The utility can be thought of as a way of ordering a two-dimensional input.

\section{Examples}

In order to demonstrate the effectiveness of our utility-based approach, we consider three networking examples through simulation.

We seek to emulate a real active probing environment where we may need to make estimates quickly, e.g. in Measurement Based Admission Control (MBAC); in this environment we can assume that network traffic rate, $\lambda$, is constant only over a small period of time, and in most cases we wish to find an estimate for this $\lambda$ quickly. We therefore assume we have a small 
amount of time $T$ to perform each experiment, and then we perform $m$ macro-replications of the experiment to assess the utility in performing the experiments at different values of $x$.

For the network under study, we fix the service rate $\mu$, arrival rate $\lambda$, and any other parameters which determine the user traffic. We must decide which possible probing rates we wish to consider, a set which we call $\mathcal{X}$, which is determined by the network under study. In experimental design, this is known as the design space.

Our procedure is:

1. Pick a probing rate $x \in \mathcal{X}$.

2. Simulate a queue running without probing to allow for a burn-in period.

3. Continue simulating the queue, but now introduce probe packets at rate $x$. Note the times spent in the system for our probe packets.

4. When the simulated queue has been running for the chosen experimental time $T$, form the estimator $\hat{\theta}$ using our choice of estimator.

5. Using the same random numbers generated for the underlying traffic arrival and service times, re-run the simulation without probes to assess the mean delay to the underlying packets caused by probing.

6. Repeat steps 2 to 5 in order to estimate (mean) bias and variance of $\hat{\theta}$, and to calculate mean delay to packets in the underlying queue. We do this $m$ times to get $m$ macro-replications. 
7. Repeat steps 2 to 6 for all $x \in \mathcal{X}$ in order to estimate bias and variance of the estimator $\hat{\lambda}$ for different values of $x$.

\subsection{Example 1: A single $\mathrm{M} / \mathrm{M} / 1$ queue}

We now choose a simple queue upon which to demonstrate the methodology, so we initially assume that our network consists of a single M/M/1 queue; this network is not intended to be representative of any network in particular, but we present it here as a simple example that the reader may be familiar with, which will enable the method to be clearly demonstrated.

For our M/M/1 queue, we performed simulations setting service rate $\mu=5$ per second $\left(s^{-1}\right)$ throughout. We assume here that we wish to estimate the arrival rate, which we call $\theta$. Any reasonable estimator may be picked, and different estimators will in general produce different estimates and thus different optimal rates. Following Aigner[28], we picked an estimator

$$
\hat{\theta}=\frac{\frac{1}{N} \sum_{i=1}^{N} Y_{i}-\frac{1}{\mu}}{\frac{1}{N} \sum_{i=1}^{N} Y_{i} \frac{1}{\mu}}
$$

although we stress that the estimator is only used to provide an example of how our method might work to determine an optimal probing rate, and not to assess the quality of the estimator.

For simulation purposes, we set $\lambda=2.5 \mathrm{~s}^{-1}$. The reader will note that $\theta=\lambda$ here, i.e. we wish to measure one of the simulation parameters, but we do not use knowledge of this $\lambda$ when estimating $\hat{\theta}$ using equation (7). 
We allowed candidate points $x$ to be in the range 0.1 to 2.4 seconds, at intervals of 0.1 . The range was thus restricted such that $\lambda+x<\mu$ as studying queues at or above full load is not considered as there are no timestable distributions, and the situation is generally not of engineering interest. We allowed a burn-in period of 10 seconds. After this burn-in, we assumed that we were able to perform the probing experiment for $T=10$ seconds. $^{1}$ We performed $m=1000$ simulations (macro-replications) for each candidate point, and by looking at the $1000 \hat{\lambda}$ generated for each candidate point, were able to estimate the bias and variance of $\hat{\lambda}$. Knowing for the simulation the underlying virtual system time process $S(t)$, and the altered process after probing $S^{*}(t)$, we were able to estimate the value of our disruption function $D(x)$, letting $c(z)=z$. In other words we penalize each packet delay "linearly". To illustrate a possible utility function, we set $\kappa=\frac{1}{2}$.

\subsubsection{Results}

The results are displayed as Figure 3. The optimal probing rate is shown as the minimum on the (bottom right) graph, here when $x \approx 1.2 \mathrm{~s}^{-1}$. (We plot $-\log (\psi(-x))$ on the $\mathrm{y}$-axis since $\psi(x)$ is strongly negative for low $x$, and we wish to plot on a scale where identifying the optimum $x$ is clear. )

Noting the log scale of the $y$-axis, we see that a low rate $\left(x<0.5 s^{-1}\right)$ gives significantly worse results (lower utility) than probing at a rate $x>0.5 s^{-1}$. This means in an engineering context that, if we were to have to choose one or

\footnotetext{
${ }^{1}$ We disregarded any probe packets that had not completed service after 10 seconds.
} 

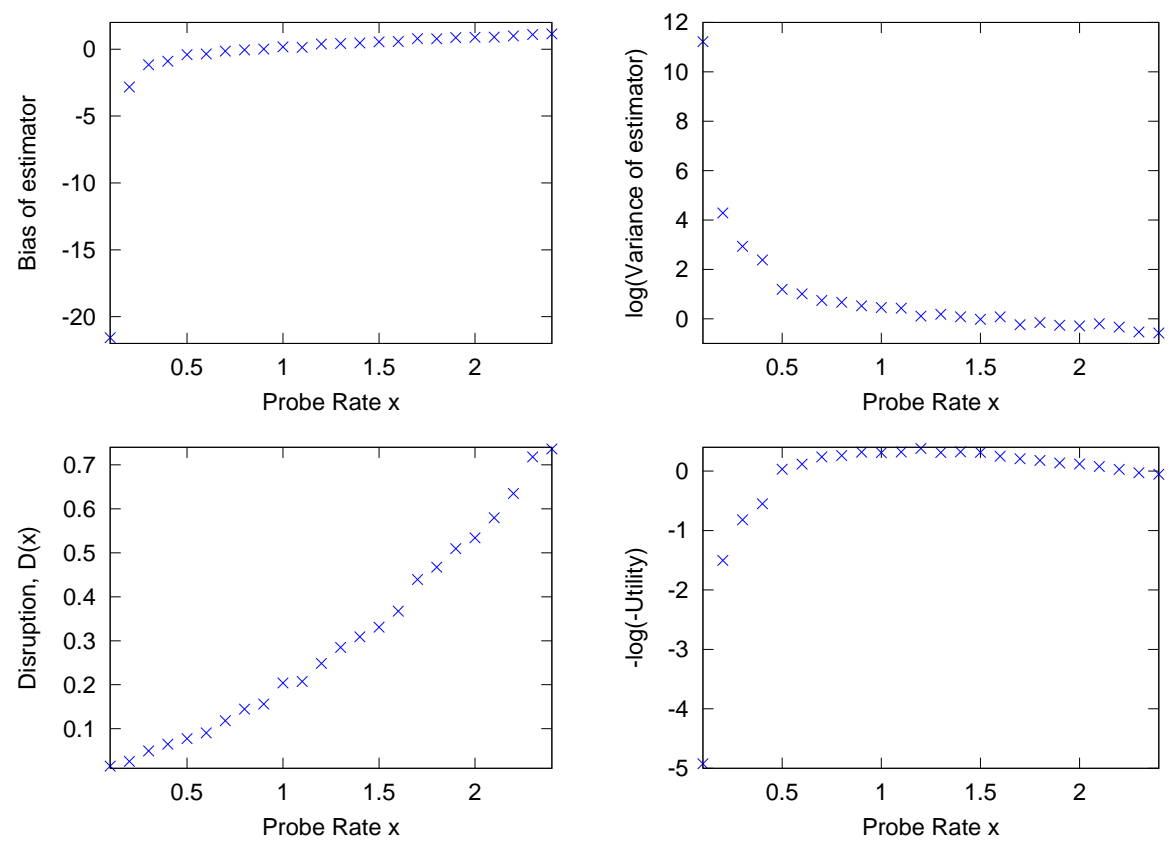

Figure 3: Building up a utility function for an $\mathrm{M} / \mathrm{M} / 1$ queue: Estimates of $\operatorname{Bias}(\hat{\lambda}), \operatorname{Var}(\hat{\lambda})$, disruption $(D(x))$ and utility $(-\log (-\psi(x)))$ all against $x$. 
the other, it appears to be substantially better to probe slightly too fast than too slow, at least in this example for an $\mathrm{M} / \mathrm{M} / 1$ queue. This poor behavior at low $x$ seems to be due primarily to a very high bias in the estimator for low $x$, as indicated by the top left graph in Figure 3. As we have limited the experiment to 10 seconds, the number of experimental Poisson probes released in this time for low rate $x$ is typically very small: e.g. for $x=0.1$ we would only expect one probe packet. As we have such a small number of probes, this seems to lead to very poor estimates.

As expected, the estimator $\hat{\lambda}$ is biased for both small and large probe rates $x$; this is a good demonstration of the research referred to in section 2.1, particularly Aigner[28], which tells us it is difficult to find an estimator with good properties for all networks and probing rates. His research shows that the best estimator depends on the design, whereas we show here that the optimal design depends on the estimator. The increasing bias may also show us a difficulty with active probing, that probes interfere with themselves, as discussed in section 2.3.

\subsubsection{Varying the utility function}

As noted above, the optimal probing rate depends heavily on the utility function chosen. We first look at varying the parameter $r$ in equation (4). The results for our disruption function and the utility function are presented as Figure 4 (changing the value of $r$ does not alter our bias or variance).

As discussed earlier, our index $r$ penalizes jitter, with higher $r$ penalizing 

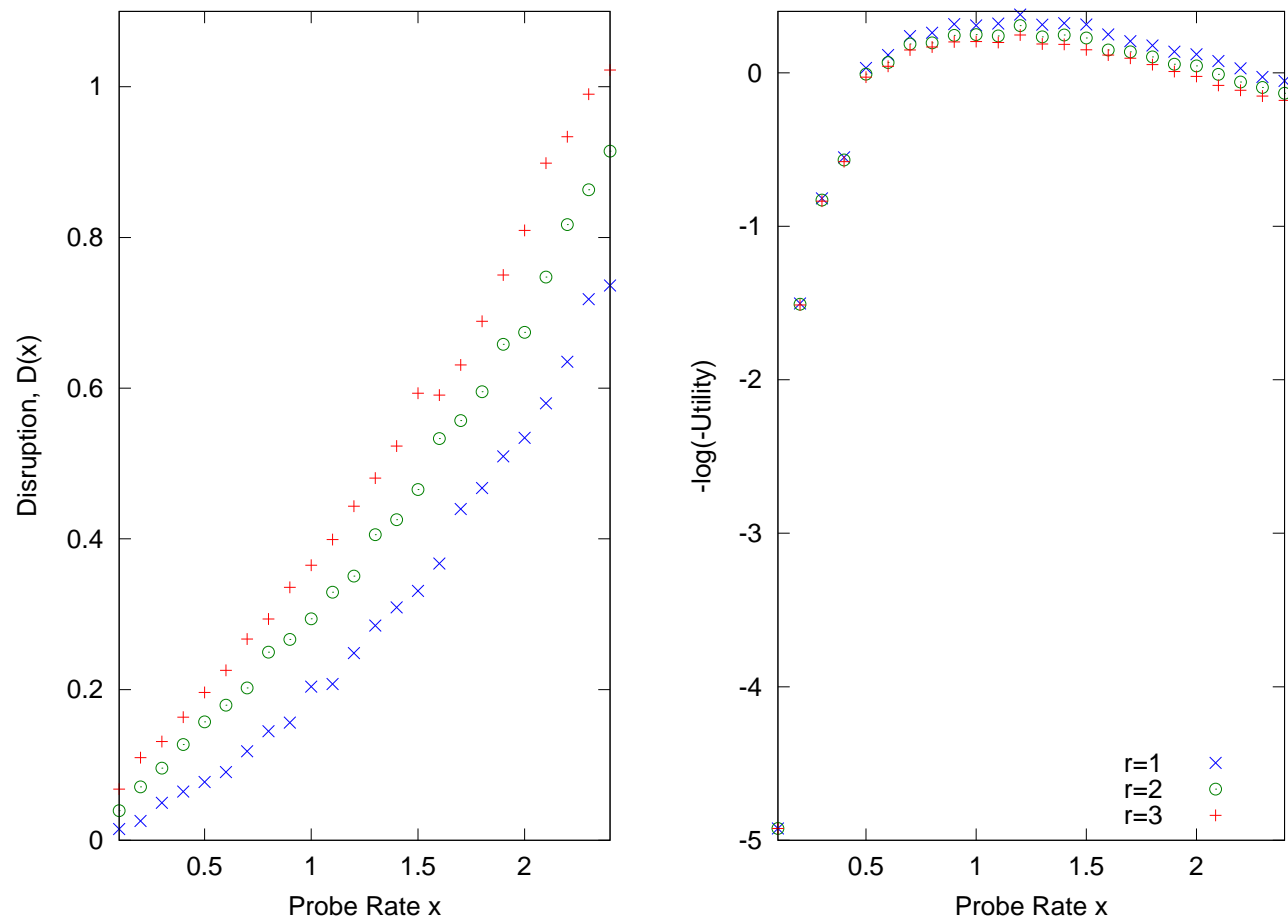

Figure 4: Varying $r$ : Disruption $(D(x))$ and utility $-\log (\psi(-x)$ for different probe rates for $r=1,2$, and 3 


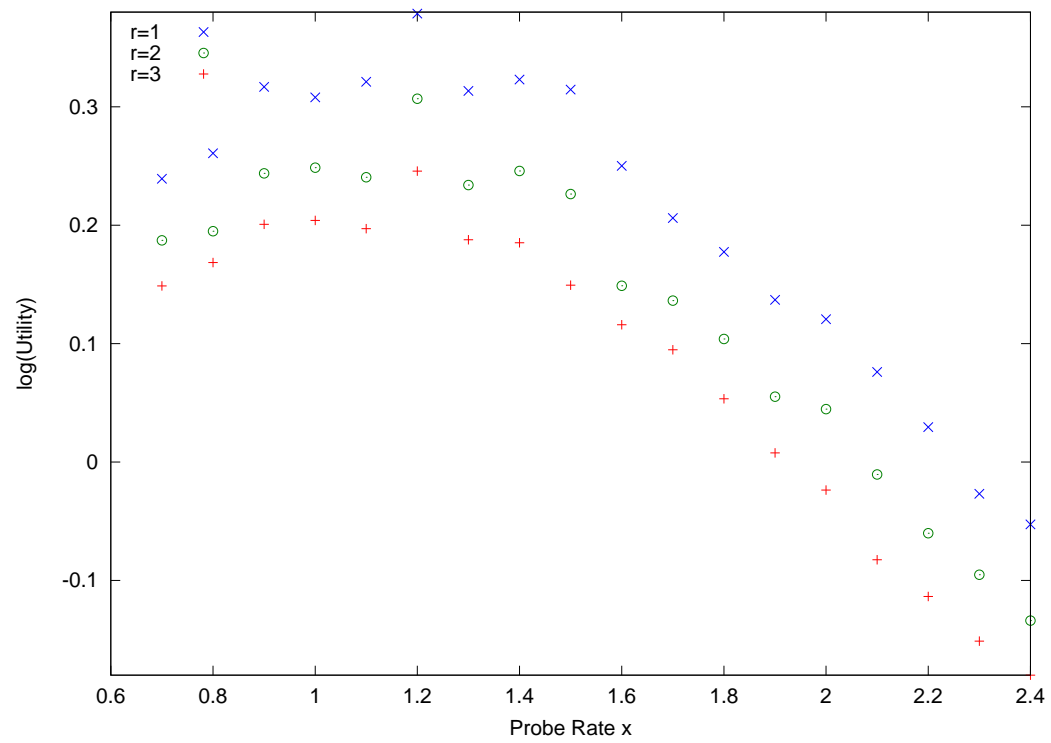

Figure 5: Varying $r$ detail: Utility $(-\log (\psi(-x)))$ for different probe rates for $r=1,2$, and $3, \kappa=0.5$

large jitter more. As we expect, higher probe rates are now penalized as jitter increases slightly as the network load increases. Figure 5 shows more detail on how utility varies with the jitter-penalizing parameter $r$; we look here only at the higher probe rates $(x \geq 0.6)$ where we are likely to find a high utility and therefore an optimal probe rate. We see that the value of $r$ does not make a substantial difference. The relative merits of different probing rates, and the optimal probing rate for all $r=1,2,3$ from these simulated data is $x=1.2 s^{-1}$. Note that the absolute value of utility, i.e. the heights of the graph, are not important in this application, as we seek only to find the optimal $x$ for a fixed value of $r$.

Figure 6 shows the changes in the utility when we set $\kappa=0.05$. This 


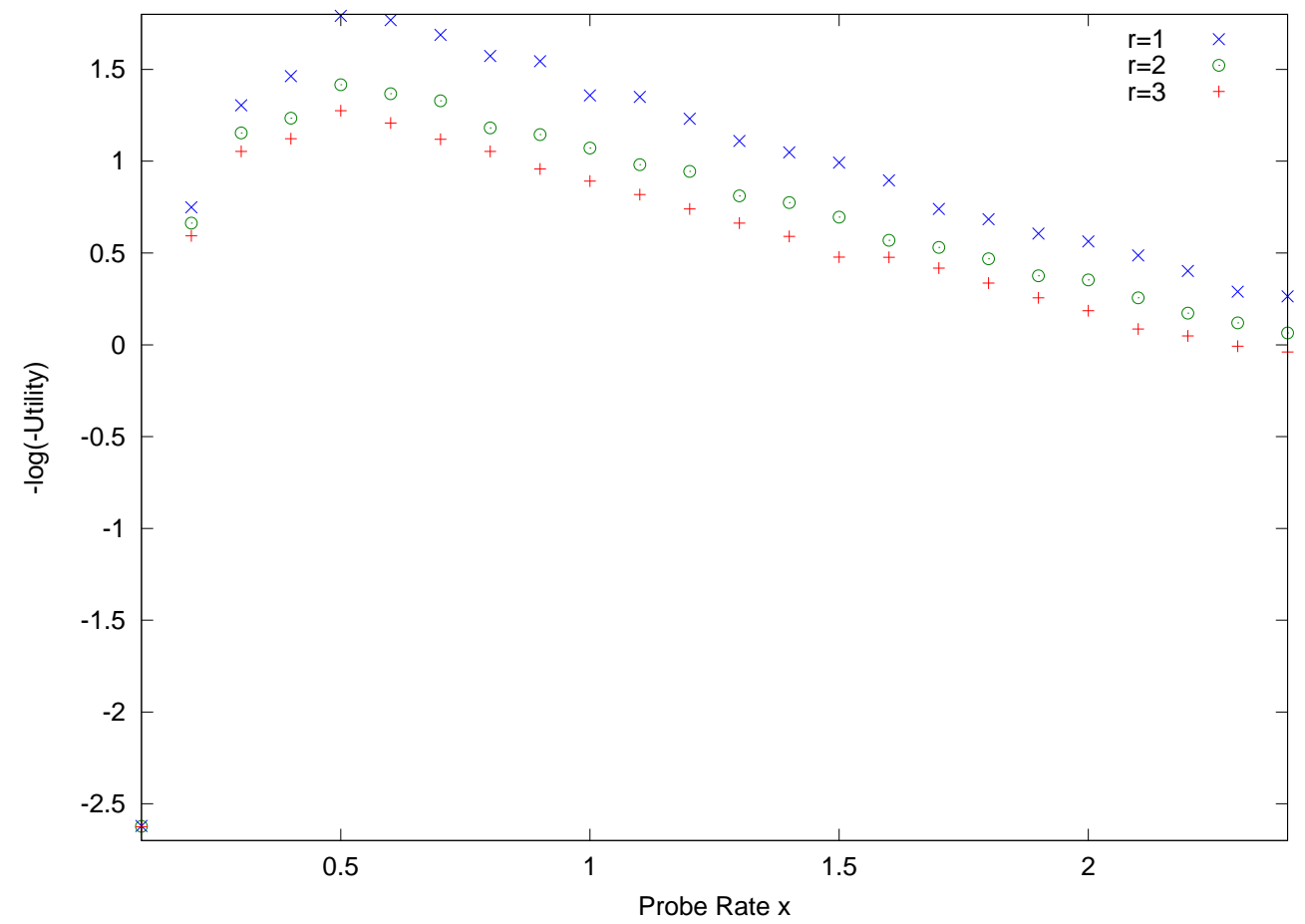

Figure 6: Varying $k$ : Utility $(-\log (\psi(-x)))$ for different probe rates for $r=1,2$, and $3, \kappa=0.05$ 
change in the value of $\kappa$ could represent a network engineer now being more concerned with minimizing delays in the network as opposed to minimizing the mean square error of our estimator. We see here that the optimal probing rate is lower, and can again be read as the maximal points of the three data sets, here $x \approx 0.5 s^{-1}$ for all $r$.

\subsection{Example 2: Estimating packet loss probability in a VoIP system}

We now demonstrate the usefulness of our methodology on a more realistic model. We assume we have a VoIP PBX with 80 users which connects via a router to a network. We know the buffer capacity of the router, and wish to measure the probability that a packet is dropped in the network.

For demonstration purposes, we simulate this model by 80 on-off sources representing the users. Each user talks (is active) for a period which is exponentially distributed with period $T_{\mathrm{on}}$, and then is silent for a period exponentially distributed with period $T_{\text {off }}$. When active, the users generate 167 VoIP packets per second, and we let the size of the packet generated be 53 bytes. We assume that the line has a capacity of $2.5 \mathrm{Mbps}\left(2.5^{*} 10^{6} \mathrm{bps}\right)$. We initially assume our VoIP PBX has a buffer capacity of 100 packets, and if an extra packet arrives while the buffer is full it will be discarded.

Let us assume we wish to estimate the probability that a cross-traffic packet is discarded, which we call $\theta$. We do this by sending probes into the 
network at a uniform rate $x$, and for this example we allow probing at integer rates up to 100 probes per second, so our design space $\mathcal{X}=\{i \in \mathcal{N} ; i \leq 100\}$. We assume the probes are the same size as the VoIP user traffic (53B).

Crucially, we must now decide on the form of our utility function. For a VoIP conversation, we can afford a delay of a certain amount per packet before that delay affects the quality of the conversation. The level of the delay will vary according to, for example, the number of network hops between those conversing, and the network equipment in between, but in general a recommended delay budget is prescribed for each hop. Let us assume here that we can tolerate up to a $10 \mathrm{~ms}$ delay, so our cost function becomes

$$
c(x)= \begin{cases}1 & \text { if } x \geq 0.01 \\ 0 & \text { otherwise }\end{cases}
$$

For VoIP networks, we know that jitter is a particularly undesirable phenomenon, so let us set our penalizing parameter $r=2$ to penalize this.

In forming our utility function, let us assume that these are important users. Whilst we wish to monitor the loss in the network, user experience is important, so we set our constant $\kappa=0.1$ to express the relative weights between disrupting of the network and measurement success.

We use our procedure described above in section 4 to again try to find an optimal probing rate. We allow a burn-in period of 10 seconds. Our experimental time $T$ is also 10 seconds. We use the simple estimator $\hat{\theta}=$ $\frac{\text { (Number of probe packets lost) }}{\text { (Total number of probes sent) }}$. We perform $m=1000$ macro replications 
for each candidate design point (probing rate).

\subsubsection{Results}
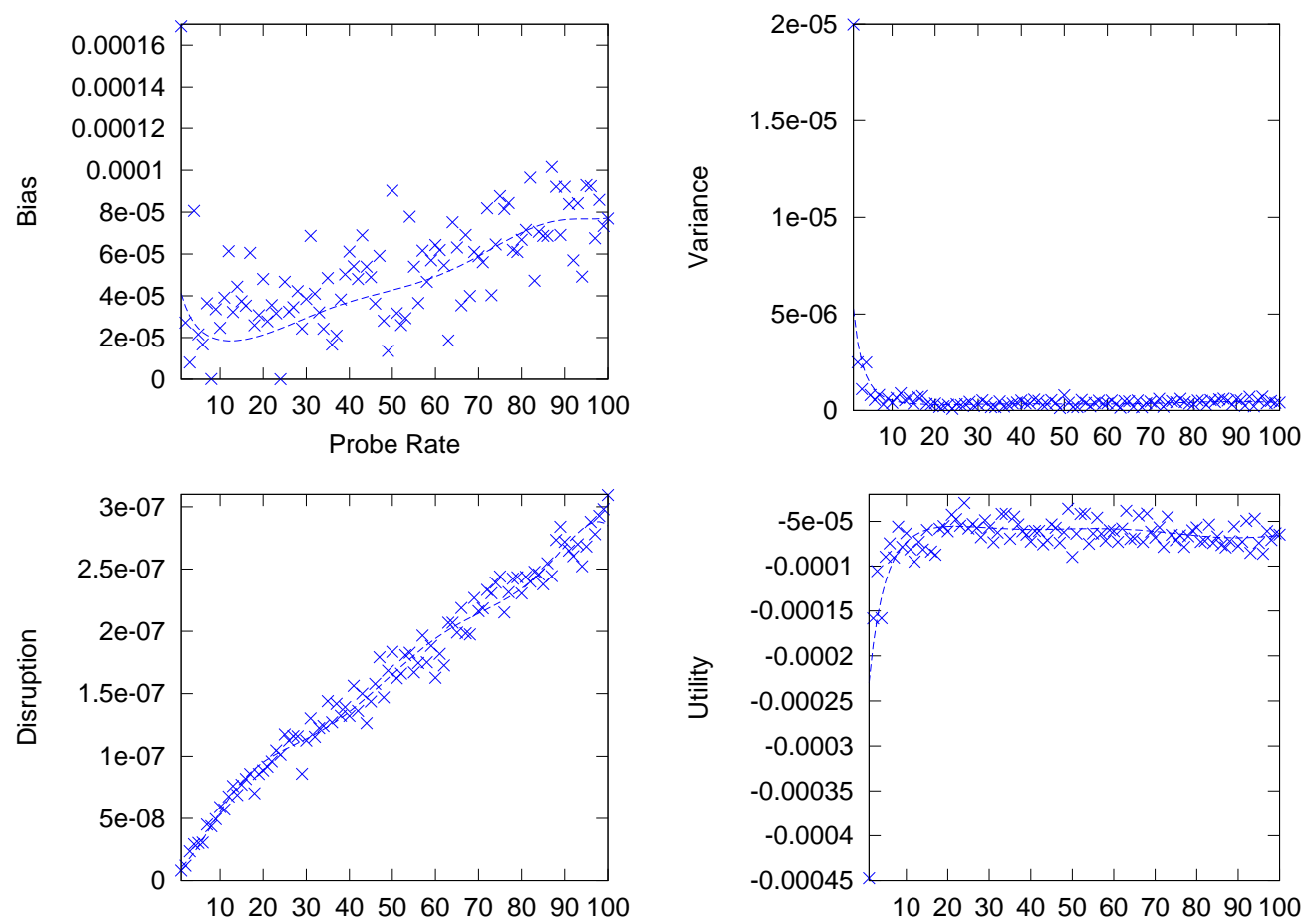

Figure 7: Bias, Variance, Disruption, and Utility $(\psi(x))$ for different probe rates for estimating packet loss percentage

The results are presented as Figure 7 . We see readily once again that, in general, bias and disruption tend to be smaller for small probe rates. There is some variance in these simulated data, so we have fitted a sixth order polynomial regression to the log transformed data for each of bias, variance, disruption, and utility, and shown this as a line in each of the graphs.

It seems here that, for high probing rates, putting in more probes increases 
the variance. As discussed, we might expect to see more measurements mean less variance, but here evidently the increased probing rate means that the variation in packet loss between our simulations is increased. In other words, probing not only alters the packet loss (bias increase), but also means that this packet loss is more variable.

We remember that the bias, variance, and disruption are only estimates we gather from the data; we do not know their true values. We see that very low rates (less than 5 probes per second) produce much worse results than results in the range 5 to 100 probes per second. The very best results (seen from the maximum of the line of best fit) were achieved probing at around 20

probes per second, and the utility decreases slightly when further increasing the probe rate. In practice, we might recommend that probing at any rate between 5 and 100 provides results with approximately the same utility.

\subsection{Example 3: Probing for available bandwidth in a network}

We now demonstrate how our utility function applies to measuring for available bandwidth. We use the same network setup of 80 voice sources as described above, however we now assume that we are interested in measuring the available bandwidth in the network. As we know the capacity of the network (2.5Mbps), estimating available bandwidth is equivalent to estimating the traffic rate. 
Recent research has focused on packet pair probing as a good way of measuring available bandwidth; by allowing two probe packets to enter a network such that one immediately follows the other, by noting any increased difference in their exit times we can make an estimate of the amount of crosstraffic that must have occurred between the entry of the first packet and that of the second. If we know the capacity of the network, and the amount of user traffic, we can easily subtract the latter from the former to gain the available capacity.

Indeed, allowing packet triples, quadruples, or even longer packet trains is currently the subject of research. We choose here to demonstrate our method with packet pairs, as the principle is the same.

Keeping the same simulation of user traffic as in example 2 above, we now allow our probing regime to consist of sending $x$ pairs of probes per second, and we allow $x$ to vary. We are now interested in estimating the cross-traffic rate which we now call $\theta$. Given the probe size $P=53$ Bytes, the service rate $\mu=2 \times 10^{6} \mathrm{bps}$, and measuring $\Delta$, the difference in time between probe pairs leaving the system, we estimate $\theta$ using the estimator

$$
\hat{\theta}=\frac{\left(\Delta-\frac{P}{\mu}\right) \mu+P}{\frac{P}{\mu}} .
$$

We set our jitter parameter $r=1$ and our weighting parameter $\kappa=0.1$.

We plot the results as Figure 8, here fitting a sixth order regression to the untransformed data for each variate and showing this as a line on each graph. 

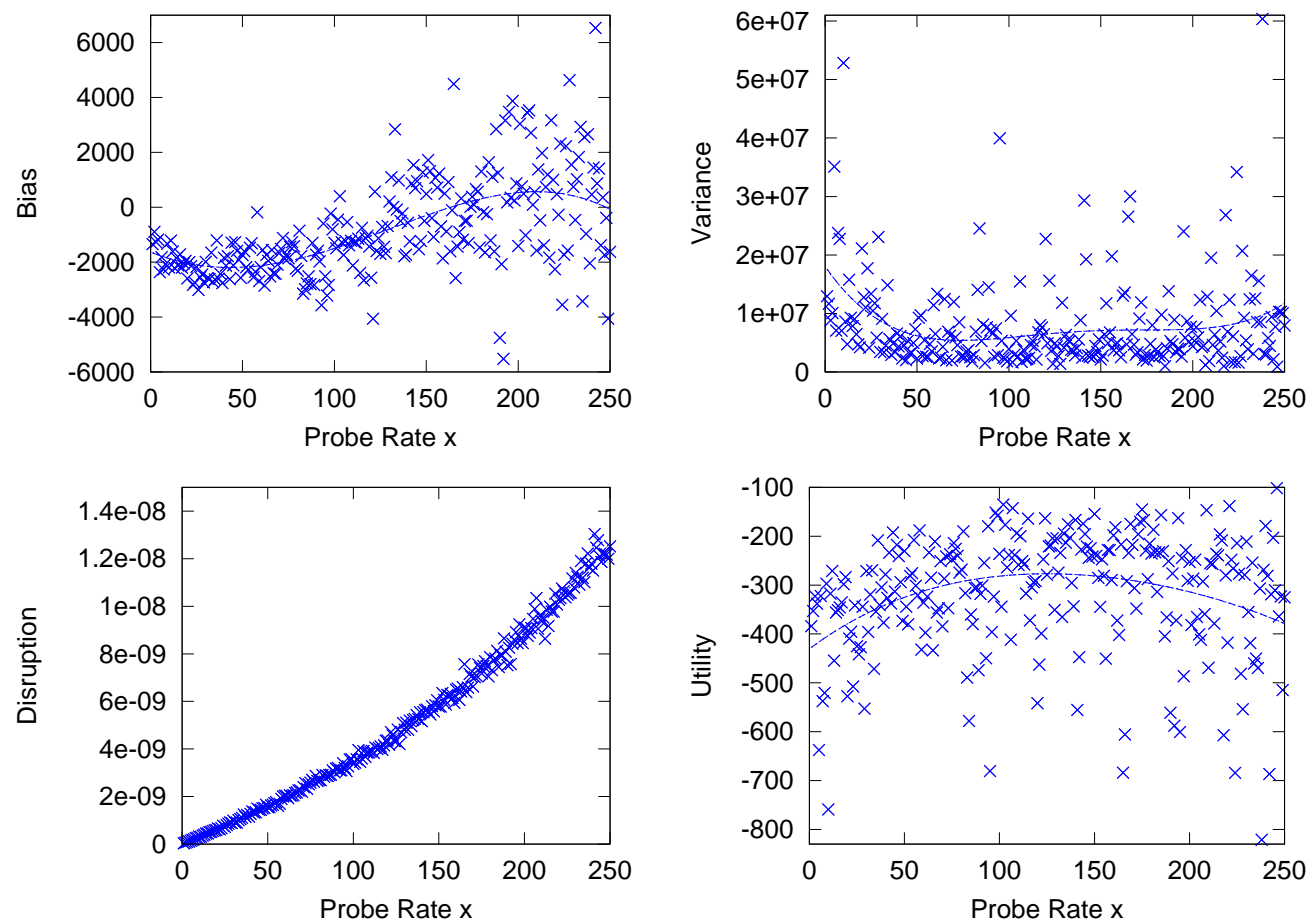

Figure 8: Bias, Variance, Disruption, and Utility $(\psi(x))$ for different probe rates (pairs per second) for estimating available bandwidth 
It is worth noting that here the bias is typically around $0.1 \%$ of the true value of available bandwidth, due to the way the paired probing mechanism is constructed. It is again evident that the utility for this estimator is lower for low probing rates, and that a moderate probing rate may be better. It looks as though probe rates of around 100-150 packet pairs per second seem best for this utility function.

\subsection{Practical considerations}

\subsubsection{Finding bias and disruption offline in a real network}

We have demonstrated that, given full knowledge of a specific network, we can determine the optimal probing rate for our network. In practice we may not have full knowledge of the network; in particular, when building up our utility function, we have assumed that both our bias and our disruption function $D(x)$ are known, but in practice we cannot determine them.

Dealing with bias is not a problem unique to measuring data networks, although seems often to be ignored in the network measurement literature; it is a fundamental problem when using active measurement of data networks that there must be a bias introduced between measurement of the "ground truth" and the altered network that is formed when we add active probes to the network. For some discussion of this, we refer the reader to Roughan [21]. Although Roughan considers variance, there are similar practical difficulties when trying to determine the disruption function. 
One possible approach to estimating bias and disruption would be by use of simulation. We build a simulator of the real network "off line" and determine the optimal probing rate using the techniques described in this paper for use with the real network. For the simulator, it is possible to see how the network responds with and without the active probes, and thus estimate the bias and disruption functions, from which we can find an optimal probing rate. Although our optimal probing rate would be exact for the simulated model, discrepancies between the model and the real network would in general mean that an optimal rate for the model was sub-optimal for the emulator. In some situations, however, this may be the best we can do; approximating complicated networks by simpler networks (or queues) is not uncommon in seeking tractable solutions to measuring complex systems. We plan to assess in future work how the model discrepancy between real network and simulator affects the success of measurement.

\subsubsection{Relaxing assumptions used in examples}

In our examples we have explicitly or implicitly made some assumptions: stationarity of the network, i.e. that the arrival probability of traffic is constant with time; simplified networks which exhibit properties such as First Come, First Served (FCFS); lack of any routing in the network. The procedure outlined in this paper to find the optimal probing rate is identical whether these assumptions are used or not, however of course the response for bias, disruption, and in particular variance might change dramatically if we relax 
these assumptions. We picked these networks as they are simple networks which the reader may be familiar with, and we are able to readily simulate them in order to estimate disruption and bias.

\section{Conclusions}

In [5], we demonstrated how to approximate a queue by a Markov chain model, taking into account interference caused by active probing, and found an exact optimal design for this model: an exactly optimal design for a (potentially) inexact model. In this paper, the optimal design (optimal probing

rate) is found approximately in the sense that we calculate bias, variance, and disruption from estimates derived from a simulation.

This method is flexible. We need know very little about the network, or use any theoretical queueing models in determining an optimal probing rate. We simply need to quantify what is important to us in measurement, for example how accurate and precise we wish our network measurement to be, at the expense of possibly disrupting the network. We have demonstrated our method for several networks, and our results show clearly that optimal rates can be found in this way for any network under consideration.

As in many other applications of statistics, expert knowledge must be used to quantify the constants used in the utility function; the practitioner must be able to quantify the relative effect of mean delay, and variance in delay (jitter) compared with measurement accuracy and disruption to the network. 
We feel this subjectivity is a valuable tool in asking network practitioners to think about their aims and targets for successful measurement. Indeed, in many applications in network monitoring and control, success often has a more tangible utility: whether the network meets a service level agreement (SLA), and what financial penalties failure to meet this might entail. The utility-based approach outlined in this paper could be used in conjunction with an SLA monitoring system.

\section{Acknowledgment}

This work was supported by EPSRC Grant EP/G012628/1: "Optimal design of performance measurement experiments for complex, large-scale networks".

\section{References}

[1] L. Chen, T. Sun, G. Yang, M. Sanadidi, M. Gerla, Ad hoc probe: path capacity probing in wireless ad hoc networks, in: First International Conference on Wireless Internet, 2005. Proceedings, 2005, pp. 156-163.

[2] Y. Su, R. Wu, R. Li, W. Duan, J. Luo, A Novel Measurement-Based Call Admission Control Algorithm for Wireless Mobility Networks under Practical Mobility Model, in: 2010 International Conference on Communications and Mobile Computing, IEEE, 2010, pp. 364-368. 
[3] Y. Na, S. Jun, C. Yuxin, J. Ye, Measurement-Based Admission Control Algorithm for Wireless IP Network, in: WRI International Conference on Communications and Mobile Computing, 2009. CMC'09, Vol. 2, 2009 .

[4] A. Atkinson, A. Donev, R. Tobias, Optimum Experimental Designs, with SAS, Oxford University Press, 2007.

[5] B.M.Parker, S.G.Gilmour, J. Schormans, Measurement of packet loss probability by optimal design of packet probing experiments, IET Communications Special Edition on Simulation, Analysis and Measurement of Broadband Network Traffic 3 (6) (2009) 979-991.

URL DigitalObjectIdentifier10.1049/iet-com.2008.0075

[6] A. Clarke, Maximum likelihood estimates in a simple queue, The Annals of Mathematical Statistics 28 (4) (1957) 1036-1040.

[7] J. Jenkins, The relative efficiency of direct and maximum likelihood estimates of mean waiting time in the simple queue $M / M / 1$, Journal of Applied Probability 9 (2) (1972) 396-403.

[8] D. Aigner, Parameter estimation from cross-sectional observations on an elementary queuing system, Operations Research (1974) 422-428.

[9] J. Reynolds, The covariance structure of queues and related processes: A survey of recent work, Advances in Applied Probability 7 (2) (Jun., 1975) 383-415. 
[10] I. Basawa, N. Prabhu, Estimation in single server queues, Naval Research Logistics Quarterly 28(3).

[11] P. Billingsley, Statistical Inference for Markov Processes, The University of Chicago Press, 1962.

[12] I. Basawa, N. Prabhu, Large sample inference from single server queues, Queueing Systems Theory and Applications 3 (4) (1988) 289-304. doi:http://dx.doi.org/10.1007/BF01157852.

[13] S. Acharya, On normal approximation for maximum likelihood estimation from single server queues, Queueing Systems 313. doi:10.1023/A:1019158230616.

URL http://www. springerlink. com/content/gm1p046626630j21

[14] I. Basawa, U. Bhat, R. Lund, Maximum likelihood estimation for single server queues from waiting time data, Queueing Systems 24 (1997) 155167.

[15] M. Roughan, A Comparison of Poisson and Uniform Sampling for Active Measurements, IEEE Journal on Selected Areas in Communications 24 (12) (2006) 2299-2312.

[16] I. Basawa, U. Bhat, J. Zhou, Parameter estimation in queueing systems using partial information, Tech. rep., Ohio State University (June 2006).

[17] T. Chen, Parameter estimation for partially observed queues, IEEE Transactions on Communications 42 (9) (1994) 2730-2739. 
[18] R. Wolff, Poisson Arrivals See Time Averages, Operations Research 30 (2) (1982) 223-231.

[19] F. Baccelli, S. Machiraju, D. Veitch, J. Bolot, The role of PASTA in network measurement, ACM SIGCOMM Computer Communication Review 36 (4) (2006) 231-242.

[20] F. Baccelli, S. Machiraju, D. Veitch, J. Bolot, On optimal probing for delay and loss measurement, in: Proceedings of the 7th ACM SIGCOMM conference on Internet measurement, ACM, 2007, pp. 291-302.

[21] M. Roughan, Fundamental bounds on the accuracy of network performance measurements, Proceedings of the 2005 ACM SIGMETRICS international conference on Measurement and modeling of computer systems (2005) 253-264.

[22] F. Baccelli, S. Machiraju, D. Veitch, J. Bolot, Probing for loss: The case against probe trains, Communications Letters, IEEE (99) (2011) 13.

URL http://ieeexplore.ieee.org/xpls/abs_all.jsp?arnumber= 5738312

[23] S. Sun, W. Xiao, Optimal linear estimators for systems with multiple random measurement delays and packet dropouts, International Journal of Systems Science 1-13doi:10.1080/00207721.2011.601347. 
URL http://www.tandfonline.com/doi/abs/10.1080/00207721. 2011.601347

[24] C. L. Nicholas G. Duffield, M. Thorup, Optimal Combination Of Sampled Measurements, 2007, WO Patent WO/2007/011,947.

URL http://www . wipo.int/patentscope/search/en/w02007011947

[25] O. Yu, E. Saric, A. Li, Integrated connection-level and packet-level QoS controls over wireless mesh networks, Journal of Parallel and Distributed Computing 68 (3) (2008) 336-347.

[26] L. Takacs, Combinatorial Methods in the Theory of Stochastic Processes, John Wiley and Sons, 1967.

[27] D. Cox, V. Isham, The Virtual Waiting-Time and Related Processes, Advances in Applied Probability 18 (2) (1986) 558-573.

[28] D. Aigner, Parameter estimation from cross-sectional observations on an elementary queuing system, Operations Research 22. 